\title{
A TOPOLOGICAL CLASSIFICATION OF CERTAIN 3-MANIFOLDS
}

\author{
BY G. BURDE AND H. ZIESCHANG
}

Communicated by Eldon Dyer, May 26, 1967

In this paper we shall prove that a compact 3-manifold, which can be fibered over $S^{1}$ is topologically determined by its fundamental group and the subgroups belonging to its boundary components.

This theorem was first supposed by J. Stallings [2] and proved in the case of a closed manifold by L. Neuwirth [1]. His proof for bounded orientable manifolds is not complete.

Let $M$ be such a compact 3-manifold with boundary components $B_{1}, B_{2}, \cdots, B_{r}, r \geqq 0$. We denote by $\left[A_{i}\right]$ the class of conjugated subgroups in $\pi_{1}(M)=G$ generated by loops on $B_{i}$. We call $\left\{G,\left[A_{1}\right]\right.$, $\left.\cdots,\left[A_{r}\right]\right\}$ the peripheral system of $M$.

A fibering of $M$ over $S^{1}$ is obtained from the product $F \times I$ of a compact surface $F$ and the unit interval $I$ by identifying $(F \times 0)$ and $(F \times 1)$ by a homeomorphism $\zeta$ of $F$. The boundary components of $M$ are tori and Klein-bottles. We write $M=F \times I / \zeta$.

$\zeta$ and $\zeta^{*}$ belong to the same class $[\zeta]$ of homeomorphisms, if they are connected by the following operations:

(i) isotopic deformation

(ii) conjugation with a homeomorphism of $F$

(iii) replacing $\zeta$ by $\zeta^{-1}$.

$F \times I / \zeta$ and $F \times I / \zeta^{*}$ are homeomorphic if $\zeta$ and $\zeta^{*}$ belong to the same class. The proof is immediate (see [1]). We consider the fundamental group $N$ of $F$ as a subgroup of $G$. The projection $M \rightarrow S^{1}$ induces a homomorphism $\chi: G \rightarrow Z$ with kernel $N . \chi\left(A_{i}\right), A_{i} \in\left[A_{i}\right]$, is a subgroup of $Z$ with finite index $n_{i} . n_{i}$ is the number of boundary curves of $F$ contained in one boundary component $B_{i}$ of $M .^{1} F$ has $n=\sum_{i=1}^{r} n_{i}$ boundary curves. If $n=0$, then by $N$ the type of $F$ is given. For $n>0, N$ is a free group, and we have to decide whether $F$ is orientable or not. Let $c_{i 0}$ be some boundary curve of $F$ belonging to $B_{i}$, and choose $A_{i} \in\left[A_{i}\right]$ such that the generator of the infinite cyclic group $A_{i} \cap N$ is represented by $c_{i 0}$ resp. its inverse $c_{i 0}^{-1}$. (The trivial case where $F$ is a disk is excluded.) The cyclic groups $t^{j} A_{i} t^{-j} \subset N$, $j=1,2, \cdots, n_{i}-1$ are generated by the boundary curves $c_{i, j}^{c_{i j}}$, $j=1, \cdots, n_{i}-1, \epsilon_{i j}= \pm 1$, of $F$, contained in $B_{i}$. The loops $c_{i j}$, $i=1, \cdots, r, j=0,1, \cdots, n_{i}-1, \cdots, \epsilon_{i j}= \pm 1$, are as elements of $N$ determined modulo conjugation in $N$.

1 The case $n_{i}>1$ was not observed in [1]. 
LemMa. $F$ is orientable if and only if for some choice of the exponents $\epsilon_{i j}= \pm 1$

$$
\prod_{i=1}^{r} \prod_{j=0}^{n_{i}-1} c_{i j}^{i_{i j}} \in[N, N] .
$$

( $[N, N]$ denotes the commutator subgroup of $N$.) The proof of the lemma is postponed.

$F$ (assuming boundary curves) may now be wholly described in algebraic terms: $n=\sum_{t=1}^{r} n_{i}$ is the number of boundary curves of $F$. The lemma is a tool to decide about orientability. Knowing this, the rank of $N$ gives the genus of $F$.

We now set out to describe $\zeta$. $\zeta$ induces an automorphism $\alpha$ of $N$. The automorphism class of $\alpha$ (modulo inner automorphisms) can be represented by

$$
\alpha: a \rightarrow t a t^{-1}, \quad a \in N .
$$

Replacing $t$ by $t^{-1}$ means replacing $\alpha$ by $\alpha^{-1}$.

Theorem. Let $M=F \times I / \zeta$ be a fibered 3-manifold with peripheral system $\left\{G,\left[A_{i}\right]\right\}$, and normal subgroups $N, \chi: G \rightarrow Z, \chi^{-1}(0)=N$. Suppose, that $M^{*}$ is another irreducible manifold with peripheral system $\left\{G^{*},\left[A_{2}^{*}\right]\right\}$, and $\Phi$ an isomorphism between $G$ and $G^{*}$ mapping $\left[A_{i}\right]$ onto $\left[A_{i}^{*}\right]$. Then $M$ and $M^{*}$ are homeomorphic.

Proof. Define $N^{*}=\Phi(N)$, and $\chi^{*}: G \rightarrow Z^{*}, \chi^{*-1}(0)=N^{*}$. Then $M^{*}=F^{*} \times I / \zeta^{*}$ by Stallings theorem [2]. As the systems of groups $\left\{G, N,\left[A_{i}\right]\right\}$ and $\left\{G^{*}, N^{*},\left[A_{i}{ }^{*}\right]\right\}$ are isomorphic we can deduce

$$
n_{i}^{*}=\left[Z^{*}: \chi^{*}\left(A_{i}^{*}\right)\right]=\left[Z: \chi\left(A_{i}\right)\right]=n_{i} \text {. }
$$

By the argument given above it follows that $F$ and $F^{*}$ are homeomorphic, $\zeta^{*}$ induces the automorphism class $\left[\alpha^{*}\right]$,

$$
\begin{gathered}
\alpha^{*}: a^{*} \rightarrow t^{*} a^{*} t^{*-1}, \quad a^{*} \in N^{*}, \\
\text { or }\left[\alpha^{*-1}\right] \text {, i.e. }\left[\phi \alpha \phi^{-1}\right]=\left[\alpha^{*}\right] \text { or }\left[\phi \alpha \phi^{-1}\right]=\left[\alpha^{*-1}\right], \quad \phi=\Phi \mid N .
\end{gathered}
$$

As $\Phi$ maps the peripheral system of $M$ onto that of $M, \phi$ maps the peripheral system of $F$ onto that of $F^{*}$. We may therefore apply the Nielsen theorem for bounded surfaces [3]. It follows, that $\phi$ is induced by a homeomorphism $\eta: F \rightarrow F^{*}$.

By the Baer theorem [3] $\eta \zeta \eta^{-1}$ and $\zeta^{*}$ resp. $\zeta^{*-1}$ are isotopic, hence they belong to the same class [ $\left.\zeta^{*}\right]$. This implies $F^{*} \times I / \zeta^{*}$ is homeomorphic $F \times I / \zeta$. It remains to prove the lemma: Any compact nonorientable surface possesses a system of canonical curves $a_{1}, \cdots, a_{n}$, 
$b_{1}, \cdots, b_{m}$ with $\prod_{i} a_{i} \prod_{j} b_{j}^{2}$ homotopic to zero. We maintain that this can be achieved for any chosen orientation of the boundary curves $r_{i}$, where $s_{i} r_{i} s_{i}^{-1}$ represents $a_{i}$.

As we can permute the $a_{i}$ by braid automorphisms, it suffices to show, that $r_{n}$ can be replaced by $r_{n}{ }^{-1}: \cdots a_{n} b_{1}{ }^{2} \cdots=\cdots a_{n} b_{1} a_{n}{ }^{-1}$ $\cdot a_{n} b_{1} \cdots=b_{1}^{\prime} a_{n}{ }^{-1} b_{1}^{\prime}{ }^{-1} b_{1}^{\prime 2} \cdots=\cdots a_{n}^{\prime} b_{1}^{\prime 2} \cdots$ putting $a_{n} b_{1}=b_{1}^{\prime}$, $a_{n}^{\prime}=b_{1}^{\prime} a_{n}^{-1} b_{1}^{\prime-1}$. Obviously $a_{n}^{\prime}$ represents $s_{n}^{\prime} r_{n}{ }^{-1} s_{n}^{\prime-1}$.

But it is easily seen by abelianizing that $\prod_{i} a_{i}=\left(\prod_{j} b_{j}{ }^{2}\right)^{-1} \in[N, N]$. The other part of the lemma is trivial.

\section{REFERENCES}

1. L. Neuwirth, $A$ topological classification of certain 3 manifolds, Bull. Amer. Math. Soc. 69 (1963), 372-375.

2. J. Stallings, "On fibering certain 3-manifolds," in Topology of 3-manifolds and related topics, Prentice-Hall, Englewood Cliffs, N. J., 1962, pp. 95-100.

3. H. Zieschang, Über Automorphismen ebener diskontinuierlicher Gruppen, Math. Ann. 166 (1966), 148-167.

Mathematisches Seminar der Universität, Frankfurt am Main 